\title{
INFLAÇÃO E INCERTEZA INFLACIONÁRIA NO BRASIL
}

\author{
Adonias Evaristo da Costa Filho*
}

\begin{abstract}
Resumo
Este artigo estuda a relação entre o nível da inflação e a incerteza inflacionária no Brasil. Essa variável é obtida a partir de dois métodos. No primeiro, a incerteza inflacionária é estimada por meio de modelos GARCH. No segundo, a incerteza inflacionária é identificada como o desvio-padrão das expectativas de inflação. Testes de causalidade de Granger apontam para causalidade em ambos os sentidos entre a incerteza inflacionária e a inflação. A incerteza inflacionária é positivamente correlacionada com a inflação corrente, e Granger-causa o componente permanente da inflação (núcleo da inflação), quando mensurada pelo desvio-padrão das expectativas de inflação.
\end{abstract}

Palavras-chave: Inflação, Incerteza, Expectativas

\begin{abstract}
This paper studies the relationship between the level of inflation and inflation uncertainty in Brazil. This variable is obtained through two different methods. In the first one, inflation uncertainty is estimated with GARCH models. In the second one, inflation uncertainty is identified as the standard deviation of inflation expectations. Granger causality tests show that causality runs in both ways between inflation uncertainty and inflation. The results show that inflation uncertainty is positively correlated with current inflation, and Granger-causes the permanent component of inflation (core inflation), when measured by the standard deviation of inflation expectations.
\end{abstract}

Keywords: Inflation, Uncertainty, Expectations

JEL classification: E31, E52.

DOI: http ://dx.doi .org/10.1590/1413-8050/ea 125774

\footnotetext{
* Doutor em economia pela Universidade de Brasília. As opiniões expressas nesta publicação são exclusivamente do autor. E-mail: adoniasevaristo@hotmail.com.
} 


\section{Introdução}

O objetivo deste artigo é analisar a relação entre inflação e incerteza inflacionária no Brasil. Especificamente, procura responder se níveis maiores de inflação provocam um aumento na incerteza inflacionária. A relevância desta pesquisa advém do fato de que desde 2010 as expectativas de inflação se situam acima da meta de inflação estabelecida para o Brasil. Uma investigação empírica da relação entre inflação e incerteza inflacionária também pode, como reflexo, oferecer respostas para as consequências de uma postura mais flexível da autoridade monetária em relação à meta de inflação, no sentido de uma maior tolerância em relação aos extremos da banda em comparação com seu ponto central.

A literatura sobre inflação e incerteza inflacionária distingue a variabilidade da inflação da incerteza inflacionária. Esta é definida como a variância do componente não esperado da inflação, ou seja, das surpresas inflacionárias. O argumento básico é de que mesmo se a variância da inflação for maior, os movimentos podem ser previsíveis. Holland (1984) lista algumas hipóteses sobre a relação entre inflação, variabilidade da inflação e a incerteza inflacionária: i) um aumento da inflação leva a um aumento da variabilidade da inflação, ii) a variabilidade da inflação gera incerteza em relação ao comportamento da inflação no futuro e iii) a incerteza inflacionária acaba prejudicando a atividade econômica.

Ball \& Cecchetti (1990) minimizam a distinção entre a variabilidade da inflação e a incerteza inflacionária, entendida como a variância da inflação não esperada, encontrando que o nível da inflação tem efeitos semelhantes tanto na variabilidade da inflação quando em mudanças não esperadas da inflação.

Friedman (1976) e Okun (1971) tratam a variabilidade da inflação e a incerteza inflacionária como sinônimos. Para Friedman (1976), taxas de inflação mais elevadas tendem a ser mais variáveis e menos previsíveis, induzindo um encurtamento dos contratos e a perda de eficiência do sistema de preços, dificultando a distinção entre alterações nos preços relativos e no nível geral de preços:

$\mathrm{t}$ is unlikely that inflation would be as fully anticipated at high as at low rates of inflation (p. 278).

The most fundamental departure is that a high inflation rate is not likely to be steady during the transition decades. Rather, the higher the rate, the more variable it is likely to be (p. 279).

A second related effect of increased volatility of inflation is to render market prices a less efficient system for coordinating economic activity. The more volatile the rate of general inflation, the harder it becomes to extract the signal about relative prices from the absolute prices... (p. 281)

Sob o ponto de vista teórico, conforme apontado por Golob (1994), a transmissão da inflação para a incerteza inflacionária se dá por meio da resposta esperada da política monetária ao aumento da inflação. A incerteza inflacionária advém tanto do momento da reação da política monetária (timing) quanto das dúvidas em relação às defasagens dos efeitos da política monetária para a atividade econômica, e por último, para a inflação. Ball (1992) constrói um modelo teórico em que a inflação elevada produz incerteza em relação à política monetária. Se por um lado é desejável uma atitude desinflacionária por parte da autoridade monetária, por outro lado a resposta pode engendrar uma recessão. A alternância de poder entre formuladores com preferências distin- 
tas entre inflação e atividade provoca incerteza em relação às consequências de desinflar a economia.

Outro elo na relação em questão se refere às consequências da incerteza inflacionária, ou seja, os fatores que fazem com que a incerteza inflacionária seja prejudicial à economia. As consequências da incerteza inflacionária podem ser separadas entre as que produzem efeitos ex-ante e aquelas que produzem consequências ex-post (Golob 1994).

Dentro da primeira categoria, por um lado a incerteza inflacionária resulta em um aumento das taxas de juros de longo prazo. Adicionalmente, a incerteza inflacionária acarreta incerteza em relação ao comportamento de outras variáveis macroeconômicas, como a taxa de juros, arrecadação tributária, investimento, etc. Por último, a incerteza inflacionária consume recursos, pois os agentes econômicos devotam recursos para lidar com os riscos oriundos da incerteza inflacionária, por exemplo, em recursos gastos com as previsões de inflação.

Por sua vez, as consequências ex-post da incerteza inflacionária são definidas como aquelas que resultam da diferença entre a inflação realizada e a expectativa de inflação prévia. Quando os contratos são fixados em termos nominais, aumentos inesperados da inflação envolvem redistribuições de renda a favor dos devedores, com o oposto acontecendo no caso de reduções inesperadas da inflação. Adicionalmente, a incerteza tende a provocar um encurtamento dos contratos, com renegociações ocorrendo de forma mais frequente.

Cukierman \& Meltzer (1986) desenvolvem um modelo teórico para formalizar o argumento de que a incerteza inflacionária pode levar a um aumento da inflação. A modelagem segue a estrutura de Barro \& Gordon (1983) em que um comportamento discricionário por parte da autoridade monetária acaba levando a um viés inflacionário. A função objetivo do banco central contempla a atividade, que é estimulada por surpresas inflacionárias, e o crescimento da oferta monetária. A primeira variável entra positivamente (o banco central gosta de menos desemprego), enquanto a segunda entra negativamente, mas os pesos da função objetivo variam aleatoriamente ao longo do tempo. Como os controles dos procedimentos de política monetária são imprecisos, a informação por parte dos agentes é imperfeita, o que gera incerteza por parte do público se as mudanças na inflação se devem a alterações nos pesos da função objetivo da autoridade monetária ou erros temporários nos procedimentos de controle da política monetária.

No modelo em questão o governo acaba não escolhendo o nível mais eficiente de controle monetário, preferindo um certo grau de ambiguidade. Essa ambiguidade é benéfica para a autoridade monetária, pois permite um maior grau de controle sobre as surpresas monetárias. A incerteza gerada produz uma taxa de inflação maior, com a autoridade monetária utilizando surpresas inflacionárias para estimular a economia de forma oportunista. O modelo oferece uma justificativa teórica para a preferência das autoridades monetárias por um certo grau de segredo e ambiguidade, e também para um relação positiva entre a média e a variância da inflação.

A hipótese de que a inflação aumenta a incerteza inflacionária ficou conhecida na literatura como hipótese de Friedman-Ball. Por sua vez, a hipótese de que a incerteza inflacionária provoca um aumento da inflação ficou denominada na literatura como hipótese de Cukierman-Meltzer.

No âmbito empírico, a literatura em geral aponta para um efeito positivo do nível da inflação para a incerteza inflacionária, por exemplo: nos EUA (Ball 
\& Cecchetti (1990), Holland (1995), Golob (1994)), no Reino Unido (Kontonikas 2004), para diversos países europeus (Caporale et al. 2012) e para o G7 (Berument \& Dincer 2005, Grier \& Perry. 1998). Esta pesquisa busca respostas para a relação entre inflação e incerteza inflacionária no Brasil, e como essa relação se contrapõe às evidências empíricas encontradas para outros países.

Ball \& Cecchetti (1990) estudam a relação entre o nível da inflação e a incerteza inflacionária, encontrando que níveis mais altos da inflação resultam em efeitos maiores sobre a variância de choques permanentes na inflação, entendidos como choques na tendência inflacionária. Colocado de outra forma, o aumento do nível da inflação implica em uma maior incerteza em relação ao comportamento de longo prazo da inflação, em comparação com a incerteza inflacionária de curto prazo. Desse resultado decorre que a inflação produz custos substanciais na economia, por meio do aumento da incerteza inflacionária.

Caporale et al. (2012) estimam a incerteza inflacionária da zona do Euro por meio de um modelo AR-GARCH, e posteriormente analisam a relação entre a incerteza inflacionária e a inflação por meio de um vetor autorregressivo (VAR). O VAR estimado inclui, além da inflação e da incerteza inflacionária de estado estacionário, a taxa de desemprego, a variação do agregado monetário M3 em termos nominais, a variação da taxa de câmbio nominal e a inclinação da curva de juros, medida pela diferença entre as taxas de juros de 3 meses e de 10 anos. Em um teste de causalidade de Granger, os autores encontram que a inflação Granger-causa a incerteza inflacionária, não encontrando evidências de causalidade de Granger no sentido contrário.

Para o G-7, Berument \& Dincer (2005) encontram que em geral a inflação causa incerteza inflacionária,confirmando os resultados encontrados em estudos anteriores, porém por meio de um modelo component GARCH para modelar a inflação, que separa os componentes de curto e longo prazo da variância condicional. Também abrangendo o G-7, Grier \& Perry. (1998) mostraram que a inflação aumenta a incerteza inflacionária para todos os países do grupo, enquanto que a relação em sentido contrário vale apenas para o Japão e a França. Por sua vez, para os Estados Unidos e Alemanha os autores encontram que a incerteza inflacionária reduz a inflação, o que os autores atribuem à resposta da política monetária desses países, nos quais a autoridade monetária possui maior independência.

Para o Brasil, Issler (1991) estimou o modelo GARCH $(1,1)$ para a variância condicional da inflação, aumentada pela inflação defasada, utilizando dados mensais de 1971 até 1985 . O autor encontrou que a média da inflação afeta a variância condicional da inflação no Brasil, por meio de um teste de hipótese sobre o coeficiente da inflação defasada na equação da variância da inflação.

Outra pesquisa no mesmo sentido foi efetuada por Vale (2004), que estimou um modelo GARCH-in-mean bivariado, usando como variáveis dependentes o crescimento do produto e a inflação na equação da média. No estudo, foram utilizadas séries do índice de preços ao produtor, índice de preços ao consumidor e produção industrial, abrangendo o período de janeiro de 1975 a dezembro de 2001. As variâncias foram modeladas por meio do modelo GARCH $(1,1)$ para a matriz de variância e covariância. As medidas de incerteza então obtidas foram testadas posteriormente na equação da média. O autor encontrou que a incerteza inflacionária tem efeitos negativos no produto e positivos na inflação, conforme esperado.

Este trabalho está organizado da seguinte forma. Além desta introdução, 
a seção 2 apresenta as medidas de incerteza inflacionária utilizadas e os resultados obtidos com cada medida, e a seção 3 conclui. Por último, o apêndice apresenta os testes de raiz unitária utilizados.

\section{Medindo a incerteza inflacionária}

\subsection{Incerteza inflacionária estimada}

Nesta seção, seguimos a abordagem amplamente utilizada na literatura pertinente à relação entre inflação e incerteza inflacionária. A abordagem principal consiste na estimação da volatilidade condicional da inflação por meio de modelos da família GARCH. A série de volatilidade condicional estimada é então interpretada como uma proxy da incerteza inflacionária ${ }^{1}$.

Nessa etapa, foram utilizadas séries temporais com frequência mensal da taxa de inflação mensal medida pelo IPCA e do IPCA acumulado em 12 meses. A série da taxa de inflação mensal vai de junho de 1999 até setembro de 2013, abrangendo ao todo 172 observações, enquanto a série da taxa de inflação acumulada em 12 meses do IPCA compreende o período entre junho de 1999 e dezembro de 2013, contendo 175 observações. Dessa forma, ambas as séries abrangem o período do regime de metas para a inflação.

A Figura 1 apresenta um gráfico com ambas as séries, além de outras também utilizadas nesta pesquisa. Em um primeiro momento foram realizados testes de raiz unitária para averiguar a possibilidade das séries utilizadas não serem estacionárias. Os testes utilizados, apresentados no apêndice, rejeitaram a hipótese de raiz unitária nas séries.

A estrutura do modelo GARCH (p, q) é dada por:

$$
\begin{gathered}
\pi_{t}=\beta_{0}+\sum_{i=1}^{m} \beta_{i} \pi_{t-i}+\sum_{i=1}^{n} \theta_{i} \varepsilon_{t-i} \\
\sigma_{\varepsilon}^{2}=\alpha_{0}+\sum_{i=1}^{p} \alpha_{i} \varepsilon_{t-i}^{2}+\sum_{i=1}^{q} \gamma_{i} \sigma_{\varepsilon t-i}^{2}
\end{gathered}
$$

Em que $\pi$ é a taxa de inflação, $\varepsilon_{t}$ é o resíduo da equação da equação da média da inflação, dada pela $1^{a}$ equação, e $\sigma_{\varepsilon}^{2}$ é a variância condicional do resíduo, que usualmente é interpretada como uma proxy da incerteza inflacionária. Para garantir uma variância positiva, temos a seguinte restrição nos parâmetros: $\alpha_{0}>0, \alpha_{i}>0$ e $\gamma_{i}>0$. O modelo é uma generalização dos modelos $\mathrm{ARCH}$, e foi introduzido por Bollerslev (1986).

O primeiro passo para a estimação dos modelos foi ajustar modelos ARMA(m,n) para retirar a correlação serial da série de taxa de inflação. Em seguida o modelo GARCH foi aplicado ao resíduo obtido pela aplicação do modelo ARMA na série da taxa de inflação. O modelo GARCH foi aplicado na série da taxa de inflação mensal e na série da taxa de inflação acumulada em 12 meses.

Para os modelos GARCH estimados, foi considerado que o termo de erro $\varepsilon_{t}$ segue uma distribuição de erros generalizada (GED). Essa distribuição tem como casos particulares a distribuição normal (caso o parâmetro da distribuição seja igual a 2) e a distribuição exponencial dupla (caso o parâmetro que

\footnotetext{
${ }^{1}$ Praticamente todos os artigos empíricos mencionados de uma forma ou de outra utilizam a abordagem GARCH: Caporale et al. (2012), Berument \& Dincer (2005), Grier \& Perry. (1998), Berument \& Dincer (2005), dentre outros
} 


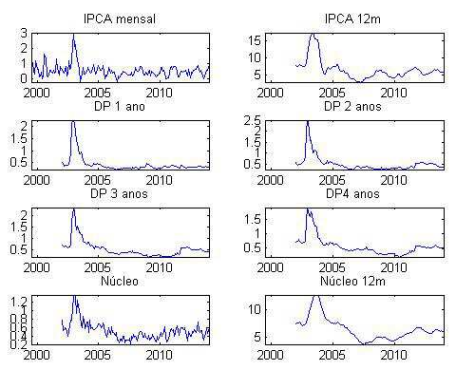

Nota: IPCA mensal, IPCA $12 \mathrm{~m}$ : IPCA acumulado em 12 meses, DP 1 ano: desvio-padrão das expectativas de inflação 1 ano à frente, DP 2 anos: desvio-padrão das expectativas de inflação 2 anos à frente, DP 3 anos: desvio-padrão das expectativas de inflação 3 anos à frente, DP 4 anos: desvio-padrão das expectativas de inflação 4 anos à frente, núcleo: núcleo mensal do IPCA médias aparadas e com suavização, núcleo $12 \mathrm{~m}$ : Núcleo do IPCA acumulado em 12 meses.

Figura 1: Dados utilizados nas estimações: IPCA mensal, IPCA acumulado em 12 meses e desvio-padrão das expectativas de inflação

governa a distribuição seja igual a 1). Adicionalmente, a estimação do parâmetro da distribuição permite obter informações sobre as caudas da distribuição. A valor estimado da distribuição GED foi inferior a 2 em todos os modelos estimados, indicando que a distribuição tem caudas mais pesadas do que a da distribuição Normal (Morettin 2006, p. 186).

Também foram estimados modelos assimétricos (E-GARCH e TARCH). Esses modelos permitem que a volatilidade responda de forma assimétrica a retornos positivos e negativos. No caso em questão, o objetivo foi testar se a incerteza inflacionária responde de forma diferente a taxas de inflação positivas ou negativas. Seguindo Morettin (2006, p. 171), a estrutura de um modelo E-GARCH(1,1) é dada por:

$$
\begin{gathered}
X_{t}=\sqrt{h_{t}} \varepsilon_{t} \\
\ln \left(h_{t}\right)=w+\beta \ln \left(h_{t-1}\right)+\alpha\left|\frac{X_{t-1}}{\sqrt{h_{t-1}}}\right|+\gamma \frac{X_{t-1}}{h_{t-1}}
\end{gathered}
$$

O componente assimétrico é dado pelo parâmetro $\gamma$. Quando $\gamma \neq 0$, a variância responde de forma diferente a choques positivos e negativos. Por sua vez, a volatilidade do modelo $\operatorname{TARCH}(1,1)$ é dada por:

$$
\begin{gathered}
\ln \left(h_{t}\right)=w+\alpha X_{t-1}^{2}+\gamma X_{t-1}^{2} d_{t-1}+\beta h_{t-1} \\
d_{t}=1, \text { se } X_{t}<0 d_{t}=0, \text { se } X_{t} \geq 0
\end{gathered}
$$

Novamente, o componente assimétrico do modelo é dado pelo parâmetro $\gamma$. 
Foram estimadas diferentes combinações de modelos $\operatorname{GARCH}(\operatorname{GARCH}(0,1)$, $\operatorname{GARCH}(1,0), \operatorname{GARCH}(1,1), \operatorname{GARCH}(2,2), \operatorname{GARCH}(2,1)$ e $\operatorname{GARCH}(1,2))$, além dos modelos assimétricos E-GARCH $(1,1)$ e $\operatorname{TARCH}(1,1)$, tanto para a taxa de inflação mensal do IPCA quanto para o IPCA acumulado em 12 meses na frequência mensal. Além de buscar obter o modelo com melhor ajuste aos dados, a estimação de diversos modelos teve o propósito de servir como exercício de robustez para os resultados obtidos neste trabalho, em particular no que concerne à causalidade no sentido de Granger entre a incerteza inflacionária e a inflação, a ser apresentada adiante.

As Tabelas 1 e 2 mostram os resultados obtidos para o IPCA mensal. A Tabela 3 mostra que para a grande maioria dos modelos as volatilidades são altamente correlacionadas ${ }^{2}$. A Tabela 4 sintetiza os principais critérios de informação (Akaike, Schwarz e Hannan-Quinn) ${ }^{3}$ dos modelos. O modelo $\operatorname{GARCH}(2,1)$ é o que resulta em maior verossimilhança e menores critérios de informação, sendo o modelo com melhor ajuste aos dados.Em todos os modelos estimados, o primeiro passo foi estimar um modelo ARMA(m,n), com o objetivo de retirar a correlação serial, para em seguida ajustar um modelo para a volatilidade condicional.

Em relação aos modelos assimétricos, muito embora o parâmetro $\gamma$ se mostre significativo no E-GARCH$(1,1)$ e no $\operatorname{TARCH}(1,1)$, o ajuste foi pior do que o observado para os modelos simétricos, no que tange à verossimilhança e aos critérios de informação.

As tabelas 5 e 6 mostram os resultados obtidos quando, em vez da taxa de inflação mensal do IPCA, os modelos são ajustados à taxa de inflação acumulada em 12 meses do IPCA, também na frequência mensal. Assim como no caso da taxa de inflação mensal do IPCA, a Tabela 7 mostra a correlação entre as volatilidades geradas por cada modelo. Para a grande maioria dos casos, as volatilidades são altamente correlacionadas. O parâmetro $\gamma$ se mostra significativo no caso dos modelos assimétricos. Considerando os valores da verossimilhança e dos critérios de informação, apresentados na Tabela 8, o modelo com melhor ajuste foi o E-GARCH $(1,1)$, seguido do $\operatorname{GARCH}(2,2)$.

As volatilidades condicionais geradas pelos diferentes modelos são apresentadas no apêndice ${ }^{4}$. Em linha com a elevada correlação entre as diferentes medidas de volatilidade, apresentadas nas Tabelas 3 e 7, os gráficos apresentam uma dinâmica comum, com o pico da incerteza inflacionária tendo sido atingido em 2003, no período considerado neste estudo.

De posse das volatilidades condicionais geradas por cada modelo, tidas como proxies da incerteza inflacionária por grande parte da literatura, o passo seguinte foi ajustar modelos VAR bivariados entre a respectiva volatilidade condicional e a taxa de inflação utilizada para gerar a série de volatilidade, i.e., a taxa de inflação mensal do IPCA ou a inflação acumulada em 12 meses do IPCA. Para selecionar as defasagens dos VARs, foram utilizados os critérios de informação (AIC, SBIC e HQIC). Como em alguns casos os VARs estimados

\footnotetext{
${ }^{2}$ Uma exceção é o $\operatorname{GARCH}(0,1)$, cujo ajuste não produz bons resultados

${ }^{3} \mathrm{O}$ critérios informação combinam a verossimilhança do modelo com o número de parâmetros do modelo. São dados por $A I C=-2 \log (L)+2 m, B I C=-2 \log (L)+m \log n$ e $H Q I C=$ $-2 \log (L)+2 \ln (\ln (n)) k) m$, onde L é a verossimilhança do modelo, m sao os números de parâmetros e n é o número de observações. Queremos encontrar o modelo com o menor critério de informação.

${ }^{4}$ Apesar de alguns coeficientes estimados terem sinal negativo, a inspeção das séries das variâncias condicionais mostra que elas são positivas ao longo de todo o período.
} 
Tabela 1: IPCA mensal

\begin{tabular}{|c|c|c|c|c|c|c|c|c|}
\hline \multirow[b]{2}{*}{ Variável } & \multicolumn{2}{|c|}{ GARCH $(1,0)$} & \multicolumn{2}{|c|}{$\operatorname{GARCH}(0,1)$} & \multicolumn{2}{|c|}{$\operatorname{GARCH}(1,1)$} & \multicolumn{2}{|c|}{$\operatorname{GARCH}(2,2)$} \\
\hline & Coeficiente & P-valor & Coeficiente & P-valor & Coeficiente & P-valor & Coeficiente & P-valor \\
\hline \multicolumn{9}{|c|}{ Equação da média } \\
\hline Constante & $0.46^{* * *}$ & 0.00 & $0.47^{* * *}$ & 0.00 & $0.43^{* * *}$ & 0.00 & $0.46^{* * *}$ & 0.00 \\
\hline$\pi_{t-1}$ & $0.49^{* * *}$ & 0.00 & $0.32^{* * *}$ & 0.00 & $0.54^{* * *}$ & 0.00 & $0.62^{* * *}$ & 0.00 \\
\hline$\pi_{t-2}$ & $0.12^{*}$ & 0.09 & $-0.21^{* * *}$ & 0.00 & & & & \\
\hline$\pi_{t-3}$ & $0.11^{* * *}$ & 0.00 & $0.12^{* * *}$ & 0.00 & $0.07^{* * *}$ & 0.06 & $0.14^{* * *}$ & 0.00 \\
\hline$\pi_{t-7}$ & $-0.25^{* * *}$ & 0.00 & $-0.22^{* * *}$ & 0.00 & $-0.23^{* * *}$ & 0.00 & $-0.17^{* * *}$ & 0.00 \\
\hline$\pi_{t-9}$ & $0.15^{* * *}$ & 0.00 & $0.16^{* * *}$ & 0.00 & $0.13^{* * *}$ & 0.00 & $0.09^{* *}$ & 0.02 \\
\hline$\pi_{t-12}$ & $0.11^{* * *}$ & 0.00 & & & & & & \\
\hline$\epsilon_{t-1}$ & $0.15 * *$ & 0.01 & $0.26^{* *}$ & 0.01 & $0.16^{* *}$ & 0.03 & & \\
\hline$\epsilon_{t-8}$ & $0.09^{* *}$ & 0.01 & $0.10^{* * *}$ & 0.00 & & & & \\
\hline$\epsilon_{t-12}$ & $0.20^{* * *}$ & 0.00 & $0.19^{* * *}$ & 0.00 & $0.27^{* * *}$ & 0.00 & & \\
\hline \multicolumn{9}{|c|}{ Equação da variância } \\
\hline Constante & $0.05^{* * *}$ & 0.00 & 0.07 & 0.86 & $0.03^{* *}$ & 0.02 & $0.02^{* * *}$ & 0.00 \\
\hline$\epsilon_{t-1} 2$ & 0.07 & 0.43 & $0.41^{* *}$ & 0.04 & $0.35^{* * *}$ & 0.01 & & \\
\hline$\epsilon_{t-2} 2$ & $0.59^{* * *}$ & 0.00 & & & & & & \\
\hline$\sigma_{t-1}^{2}$ & -0.14 & 0.98 & 0.17 & 0.42 & -0.05 & 0.55 & & \\
\hline$\sigma_{t-2}^{2}$ & -0.14 & 0.27 & & & & & & \\
\hline Parầmetro GED & $0.84^{* * *}$ & 0.00 & $0.82^{* * *}$ & 0.00 & $0.90^{* * *}$ & 0.00 & $1.30^{* * *}$ & 0.00 \\
\hline$R^{2}$ & 0.49 & & 0.48 & & 0.50 & & 0.46 & \\
\hline Erro-padrão da regressão & 0.28 & & 0.29 & & 0.28 & & 0.29 & \\
\hline Log-verossimilhança & 14.40 & & 14.29 & & 7.86 & & 13.56 & \\
\hline Critério de Akaike & -0.04 & & -0.04 & & -0.07 & & -0.00 & \\
\hline Critério de Schwarz & 0.16 & & 0.16 & & 0.15 & & 0.24 & \\
\hline Critério de Hannan-Quinn & 0.04 & & 0.04 & & 0.02 & & 0.09 & \\
\hline Observações & 163 & & 163 & & 163 & & 160 & \\
\hline \multicolumn{9}{|c|}{ Estatistica Q de Ljung-Box para os resíduos (p-valor) } \\
\hline $\mathrm{Q}(10)$ & 0.16 & & 0.05 & & 0.15 & & 0.11 & \\
\hline $\mathrm{Q}(15)$ & 0.48 & & 0.27 & & 0.47 & & 0.1 & \\
\hline $\mathrm{Q}(20)$ & 0.71 & & 0.50 & & 0.49 & & 0.11 & \\
\hline \multicolumn{9}{|c|}{ Estatistica Q de Ljung-Box para os resíduos ao quadrado (p-valor) } \\
\hline $\mathrm{Q}(10)$ & 0.04 & & 0.37 & & 0.62 & & 0.39 & \\
\hline $\mathrm{Q}(15)$ & 0.35 & & 0.88 & & 0.46 & & 0.71 & \\
\hline $\mathrm{Q}(20)$ & 0.69 & & 0.98 & & 0.70 & & 0.61 & \\
\hline
\end{tabular}

Nota: ${ }^{* *},{ }^{* *}, \mathrm{e}^{*}$ indicam significância estatistica a $1 \%, 5 \%$ e $10 \%$, respectivamente 
Tabela 2: IPCA mensal - continuação

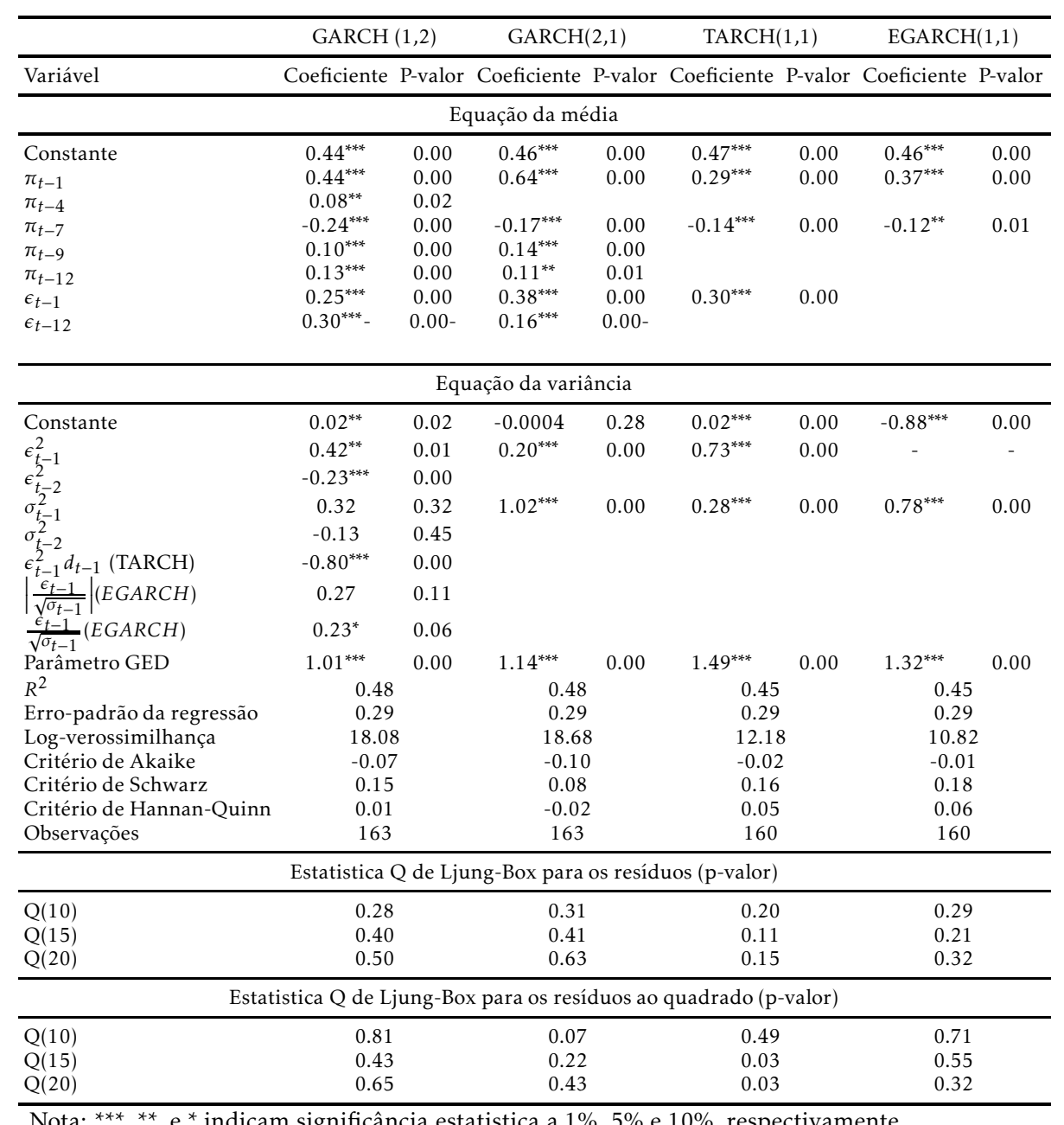


Tabela 3: IPCA mensal: Correlações entre as variâncias condicionais geradas pelos modelos

\begin{tabular}{|c|c|c|c|c|c|c|c|c|}
\hline & $\operatorname{Garch}(0,1)$ & $\operatorname{Garch}(1,0)$ & $\operatorname{Garch}(1,1)$ & $\operatorname{Garch}(1,2)$ & $\operatorname{Garch}(2,1)$ & $\operatorname{Garch}(2,2)$ & $\operatorname{EGarch}(1,1)$ & $\operatorname{Tarch}(1,1)$ \\
\hline $\operatorname{Garch}(0,1)$ & 1 & & & & & & & \\
\hline $\operatorname{Garch}(1,0)$ & 0 & 1 & & & & & & \\
\hline $\operatorname{Garch}(1,1)$ & $-0,01$ & 0,98 & 1 & & & & & \\
\hline $\operatorname{Garch}(1,2)$ & $-0,01$ & 0,95 & 0,99 & 1 & & & & \\
\hline $\operatorname{Garch}(2,1)$ & $-0,06$ & 0,92 & 0,9 & 0,87 & 1 & & & \\
\hline $\operatorname{Garch}(2,2)$ & 0,05 & 0,54 & 0,68 & 0,76 & 0,53 & 1 & & \\
\hline $\operatorname{EGarch}(1,1)$ & 0,09 & 0,88 & 0,93 & 0,94 & 0,85 & 0,75 & 1 & \\
\hline $\operatorname{Tarch}(1,1)$ & 0,06 & 0,91 & 0,95 & 0,96 & 0,84 & 0,71 & 0,98 & 1 \\
\hline
\end{tabular}

permaneciam com correlação serial na defasagem selecionada pelos critérios, aumentou-se o número de defasagens até a correlação serial ser eliminada do modelo. Em geral foi necessária a adição de apenas uma defasagem no VAR, além da apontada pelos critérios de informação, para eliminar a autocorrelação serial. As Tabelas 10 e 12 apresentam os testes dos multiplicadores de Lagrange nos modelos, assim como a defasagem utilizada ${ }^{5}$.

Entretanto, o teste de normalidade Jarque-Bera em geral rejeitou a hipótese de normalidade dos resíduos nos VARs estimados.

Para responder à questão colocada neste trabalho, estamos interessados principalmente na causalidade de Granger entre a volatilidade condicional do modelo (proxy estimada da incerteza inflacionária) e a taxa de inflação. Considerando um VAR bivariado conforme abaixo:

$$
\begin{aligned}
& y_{t}=a(L) y_{t-1}+b(L) x_{t-1}+\varphi_{t} \\
& x_{t}=c(L) x_{t-1}+d(L) y_{t-1}+v_{t}
\end{aligned}
$$

a variável $y_{t}$ não Granger-causa a variável $x_{t}$ se $d(L)=0$, ou seja, se podemos retirar as defasagens de $y_{t}$ da equação de $x_{t}$.

As tabelas 9 e 11 apresentam os resultados dos testes de causalidade de Granger entre a incerteza inflacionária obtida a partir dos modelos GARCH e a taxa de inflação. No caso da taxa de inflação mensal (tabela 9), com exceção do modelo GARCH $(2,2)$, os resultados mostram que a incerteza inflacionária Granger-causa a inflação, com a relação em sentido contrário também valendo, i.e, a inflação Granger-causa a incerteza inflacionária. A mesma conclusão vale quando utilizamos como medida de inflação o IPCA acumulado em 12 meses, com os resultados apresentados na Tabela 11. Nesse sentido, encontramos evidências tanto da hipótese de Friedman-Ball quanto da hipótese de Cukierman-Meltzer quando a incerteza inflacionária é mensurada por meio da volatilidade condicional dos modelos GARCH. O fato do resultado ser obtido para diferentes ajustes de modelos, e com base em diferentes medidas de inflação (IPCA mensal e acumulado em 12 meses) confere robustez aos resultados.

Além da causalidade de Granger, também estamos interessados na correlação entre as diferentes medidas de volatilidade e as medidas de inflação. A correlação permite obter pistas em relação à associação entre o nível da inflação e a incerteza inflacionária, em adição à precedência temporal dada pela

\footnotetext{
${ }^{5}$ Antes da estimação do modelo VAR envolvendo a taxa de inflação e a série de volatilidade condicional obtida pelo modelo, ambas foram submetidas aos testes de raiz unitária, que indicaram que as variáveis são estacionárias, exceto para a volatilidade condicional do $\operatorname{EGARCH}(1,1)$ da inflação acumulada em 12 meses. Os resultados dos testes estão apresentados no apêndice.
} 
Tabela 4: IPCA mensal - Critérios de seleção dos modelos

\begin{tabular}{lcccccccc}
\hline \multicolumn{1}{c}{ Garch(0,1) } & Garch(1,0) & Garch(1,1) & Garch(1,2) & Garch(2,1) & Garch(2,2) & EGarch(1,1) & Tarch(1,1) \\
\hline $\mathrm{R}^{2}$ & 0.48 & 0.49 & 0.50 & 0.48 & 0.48 & 0.46 & 0.45 & 0.45 \\
Erro-padrão da regressão & 0.29 & 0.28 & 0.28 & 0.29 & 0.29 & 0.29 & 0.29 & 0.29 \\
Log-verossimilhança & 14.29 & 14.40 & 17.86 & 18.08 & 18.68 & 13.56 & 10.82 & 12.18 \\
Critério de Akaike & -0.04 & -0.04 & -0.07 & -0.07 & -0.10 & -0.00 & -0.01 & -0.02 \\
Critério de Schwarz & 0.16 & 0.16 & 0.15 & 0.15 & 0.08 & 0.24 & 0.18 & 0.16 \\
Critério de Hannan-Quinn & 0.04 & 0.04 & 0.02 & 0.01 & -0.02 & 0.09 & 0.06 & 0.05 \\
\hline
\end{tabular}


Tabela 5: IPCA mensal - continuação

\begin{tabular}{|c|c|c|c|c|c|c|c|c|}
\hline & \multicolumn{2}{|c|}{$\operatorname{GARCH}(1,0)$} & \multicolumn{2}{|c|}{$\operatorname{GARCH}(0,1)$} & \multicolumn{2}{|c|}{$\operatorname{GARCH}(1,1)$} & \multicolumn{2}{|c|}{$\operatorname{GARCH}(2,2)$} \\
\hline Variável & Coeficiente & P-valor & Coeficiente & P-valor & Coeficiente & P-valor & Coeficiente & P-valor \\
\hline Constante & $5.64^{* * *}$ & 0.00 & $6.02^{* * *}$ & 0.00 & $5.60^{* * *}$ & 0.00 & $5.56^{* * *}$ & 0.00 \\
\hline$\pi_{t-1}$ & $1.50^{* * *}$ & 0.00 & $1.51^{* * *}$ & 0.00 & $1.53^{* * *}$ & 0.00 & $1.54^{* * *}$ & 0.00 \\
\hline$\pi_{t-2}$ & $-0.52^{* * *}$ & 0.00 & $-0.52^{* * *}$ & 0.00 & $-0.55^{* * *}$ & 0.00 & $-0.55^{* * *}$ & 0.00 \\
\hline$\pi_{t-7}$ & $-0.21^{* * *}$ & 0.00 & & & & & & \\
\hline$\epsilon_{t-1}$ & $0.16^{* * *}$ & 0.00 & & & & & & \\
\hline$\epsilon_{t-4}$ & $0.07^{* *}$ & 0.02 & $0.09^{* *}$ & 0.05 & $0.09^{* *}$ & 0.03 & & \\
\hline$\epsilon_{t-12}$ & $-0.71^{* * *}$ & 0.00 & $-0.81^{* * *}$ & 0.00 & $-0.76^{* * *}$ & 0.00 & $-0.77^{* * *}$ & 0.00 \\
\hline \multicolumn{9}{|c|}{ Equação da variância } \\
\hline Constante & $0.04^{* * *}$ & 0.00 & -0.00 & 0.56 & 0.005 & 0.20 & -0.00 & 0.20 \\
\hline$\epsilon_{t-1}^{2}$ & $0.62^{* * *}$ & 0.00 & $0.22^{* *}$ & 0.02 & 0.25 & 0.14 & - & - \\
\hline$\epsilon_{t-2}^{2-1}$ & -0.27 & 0.12 & & & & & & \\
\hline$\sigma_{t-1}^{t-2}$ & $0.99^{* * *}$ & 0.00 & $0.71^{* * *}$ & 0.00 & $1.09^{* * *}$ & 0.00 & & \\
\hline$\sigma_{t-2}^{2-1}$ & -0.08 & 0.51 & & & & & & \\
\hline Parấmetro GED & $1.09^{* * *}$ & 0.00 & $1.12^{* * *}$ & 0.00 & $1.30^{* * *}$ & 0.00 & $1.53^{* * *}$ & 0.00 \\
\hline$R^{2}$ & 0.98 & & 0.98 & & 0.98 & & 0.98 & \\
\hline Erro-padrão da regressão & 0.32 & & 0.32 & & 0.32 & & 0.31 & \\
\hline Log-verossimilhança & -11.00 & & -10.76 & & -13.6 & & -6.88 & \\
\hline Critério de Akaike & 0.23 & & 0.21 & & 0.26 & & 0.20 & \\
\hline Critério de Schwarz & 0.39 & & 0.36 & & 0.42 & & 0.40 & \\
\hline Critério de Hannan-Quinn & 0.29 & & 0.27 & & 0.32 & & 0.28 & \\
\hline Observações & 173 & & 173 & & 173 & & 173 & \\
\hline \multicolumn{9}{|c|}{ Estatistica Q de Ljung-Box para os resíduos (p-valor) } \\
\hline $\mathrm{Q}(10)$ & 0.16 & & 0.10 & & 0.30 & & 0.20 & \\
\hline $\mathrm{Q}(15)$ & 0.39 & & 0.15 & & 0.42 & & 0.38 & \\
\hline $\mathrm{Q}(20)$ & 0.24 & & 0.18 & & 0.61 & & 0.53 & \\
\hline \multicolumn{9}{|c|}{ Estatistica Q de Ljung-Box para os resíduos ao quadrado ( $\mathrm{p}$-valor) } \\
\hline$\overline{Q(10)}$ & 0.004 & & 0.22 & & 0.62 & & 0.22 & \\
\hline $\mathrm{Q}(15)$ & 0.04 & & 0.51 & & 0.92 & & 0.51 & \\
\hline $\mathrm{Q}(20)$ & 0.16 & & 0.74 & & 0.98 & & 0.69 & \\
\hline
\end{tabular}

Nota: ${ }^{* *},{ }^{* *}, \mathrm{e}^{*}$ indicam significância estatistica a $1 \%, 5 \%$ e $10 \%$, respectivamente 
Tabela 6: IPCA 12 meses - continuação

\begin{tabular}{|c|c|c|c|c|c|c|c|c|}
\hline & \multicolumn{2}{|c|}{$\operatorname{GARCH}(2,1)$} & \multicolumn{2}{|c|}{$\operatorname{GARCH}(1,2)$} & \multicolumn{2}{|c|}{$\operatorname{TARCH}(1,1)$} & \multicolumn{2}{|c|}{$\operatorname{EGARCH}(1,1)$} \\
\hline Variável & Coeficiente & P-valor & Coeficiente & P-valor & Coeficiente & P-valor & Coeficiente & P-valor \\
\hline \multicolumn{9}{|c|}{ Equação da média } \\
\hline Constante & $5.51^{* * *}$ & 0.00 & $5.58^{* * *}$ & 0.00 & $5.91^{* * *}$ & 0.00 & $6.80^{* * *}$ & 0.00 \\
\hline$\pi_{t-1}$ & $1.49^{* * *}$ & 0.00 & $1.53^{* * *}$ & 0.00 & $1.58^{* * *}$ & 0.00 & $1.70^{* * *}$ & 0.00 \\
\hline$\pi_{t-2}$ & $-0.51^{* * *}$ & 0.00 & $-0.55^{* * *}$ & 0.00 & $-0.59^{* * *}$ & 0.00 & $-0.73^{* * *}$ & 0.00 \\
\hline$\pi_{t-7}$ & $0.02^{* *}$ & 0.03 & & & & & & \\
\hline$\epsilon_{t-2}$ & $-0.20^{* * *}$ & 0.00 & & & & & & \\
\hline$\epsilon_{t-4}$ & $0.08^{* *}$ & 0.07 & $0.09^{*}$ & 0.06 & $0.08^{*}$ & 0.09 & & \\
\hline$\epsilon_{t-12}$ & $-0.76^{* * *}$ & 0.00 & $-0.76^{* * *}$ & 0.00 & $-0.72^{* * *}$ & 0.00 & $-0.77^{* * *}$ & 0.00 \\
\hline \multicolumn{9}{|c|}{ Equação da variância } \\
\hline Constante & $-0.00^{*}$ & 0.09 & 0.006 & 0.31 & 0.009 & 0.06 & $-0.04^{* * *}$ & 0.00 \\
\hline$\epsilon_{t-1}^{2}$ & $0.41^{* * *}$ & 0.00 & 0.27 & 0.20 & $0.34^{* *}$ & 0.02 & - & - \\
\hline$\epsilon_{t-2}^{2}$ & $-0.42^{* * *}$ & 0.00 & & & & & & \\
\hline$\sigma_{t-1}^{2}$ & $1.01^{* * *}$ & 0.00 & 0.45 & 0.63 & $0.69^{* * *}$ & 0.00 & $0.92^{* * *}$ & 0.00 \\
\hline$\sigma_{t-2}^{2}$ & 0.21 & 0.27 & & & & & & \\
\hline$\epsilon_{t-1}^{t-2} d_{t-1}(\mathrm{TARCH})$ & $-0.37^{* * *}$ & 0.01 & & & & & & \\
\hline$\left|\frac{\epsilon_{t-1}}{\sqrt{\sigma_{t-1}}}\right|(E G A R C H)$ & $-0.23^{* * *}$ & 0.00 & & & & & & \\
\hline$\frac{\epsilon_{t-1}}{\sqrt{\sigma_{t-1}}}(E G A R C H)$ & $0.24^{* * *}$ & 0.00 & & & & & & \\
\hline Parâmetro GED & $1.48^{* * *}$ & 0.00 & $1.30^{* * *}$ & 0.00 & $1.42^{* * *}$ & 0.00 & $1.49^{* * *}$ & 0.00 \\
\hline$R^{2}$ & 0.98 & & 0.98 & & 0.98 & & 0.98 & \\
\hline Erro-padrão da regressão & 0.32 & & 0.32 & & 0.32 & & 0.31 & \\
\hline Log-verossimilhança & -8.71 & & -13.40 & & -11.68 & & 0.85 & \\
\hline Critério de Akaike & 0.21 & & 0.27 & & 0.25 & & 0.12 & \\
\hline Critério de Schwarz & 0.39 & & 0.45 & & 0.43 & & 0.32 & \\
\hline Critério de Hannan-Quinn & 0.29 & & 0.34 & & 0.32 & & 0.20 & \\
\hline Observações & 173 & & 173 & & 173 & & 168 & \\
\hline \multicolumn{9}{|c|}{ Estatistica Q de Ljung-Box para os resíduos (p-valor) } \\
\hline $\mathrm{Q}(10)$ & 0.20 & & 0.30 & & 0.30 & & 0.15 & \\
\hline$Q(15)$ & 0.37 & & 0.40 & & 0.34 & & 0.36 & \\
\hline $\mathrm{Q}(20)$ & 0.51 & & 0.61 & & 0.48 & & 0.52 & \\
\hline \multicolumn{9}{|c|}{ Estatistica Q de Ljung-Box para os resíduos ao quadrado (p-valor) } \\
\hline $\mathrm{Q}(10)$ & 0.06 & & 0.53 & & 0.62 & & 0.15 & \\
\hline $\mathrm{Q}(15)$ & 0.20 & & 0.88 & & 0.90 & & 0.40 & \\
\hline $\mathrm{Q}(20)$ & 0.39 & & 0.98 & & 0.97 & & 0.47 & \\
\hline
\end{tabular}

Nota: $* * *, * *, e^{*}$ indicam significância estatistica a $1 \%, 5 \%$ e $10 \%$, respectivamente 
Tabela 7: IPCA em 12 meses- Correlações entre as variâncias condicionais geradas pelos modelos

\begin{tabular}{|c|c|c|c|c|c|c|c|c|}
\hline & $\operatorname{Garch}(0,1)$ & $\operatorname{Garch}(1,0)$ & $\operatorname{Garch}(1,1)$ & $\operatorname{Garch}(2,2)$ & $\operatorname{Garch}(2,1)$ & $\operatorname{Garch}(1,2)$ & $\operatorname{EGarch}(1,1)$ & $\operatorname{Tarch}(1,1)$ \\
\hline $\operatorname{Garch}(0,1)$ & 1 & & & & & & & \\
\hline $\operatorname{Garch}(1,0)$ & 0,18 & 1 & & & & & & \\
\hline $\operatorname{Garch}(1,1)$ & 0,37 & 0,72 & 1 & & & & & \\
\hline $\operatorname{Garch}(2,2)$ & 0,47 & 0,94 & 0,75 & 1 & & & & \\
\hline $\operatorname{Garch}(2,1)$ & 0,33 & 0,98 & 0,73 & 0,99 & 1 & & & \\
\hline $\operatorname{Garch}(1,2)$ & 0,35 & 0,8 & 0,99 & 0,82 & 0,81 & 1 & & \\
\hline $\operatorname{EGarch}(1,1)$ & 0,45 & 0,28 & 0,56 & 0,37 & 0,33 & 0,54 & 1 & \\
\hline $\operatorname{Tarch}(1,1)$ & 0,31 & 0,75 & 0,98 & 0,77 & 0,76 & 0,97 & 0,55 & 1 \\
\hline
\end{tabular}

causalidade de Granger. A Tabela 13 apresenta os coeficientes de correlação entre as medidas de inflação e as volatilidades geradas por cada modelo. Em geral as correlações são positivas e moderadas, oscilando entre 0,4 a 0,7 , implicando em uma relação positiva entre o nível da inflação e as proxies da incerteza inflacionária mensuradas pelas volatilidades condicionais.

\subsection{Incerteza inflacionária observada}

Uma desvantagem da abordagem acima, que utiliza os modelos GARCH para analisar a relação entre inflação e incerteza inflacionária, é que esta é obtida a partir da estimação de modelos. Para superar essa limitação, empregamos nesta seção uma medida diretamente observável da incerteza inflacionária, o desvio-padrão das expectativas de inflação coletadas pela pesquisa Focus do Banco Central para 1 a 4 anos à frente. Intuitivamente, períodos de incerteza inflacionária estão relacionados com uma maior dispersão das expectativas de inflação dos agentes, equivalentes à presença de caudas pesadas na distribuição de frequência das expectativas de inflação. Semelhante medida de incerteza inflacionária também foi utilizada no estudo de Golob (1994) e Holland (1995) para os Estados Unidos.

A opção pelo desvio-padrão das expectativas de inflação se deu em função da ausência de dados públicos para as variâncias das distribuições de probabilidade individuais no Brasil. ${ }^{6}$

A série utilizada vai do final de 2001 ao final de 2013. Esse período foi utilizado porque a série de expectativas de inflação começou a ser coletada em novembro de 2001. Para exemplificar os dados utilizados, para as expectativas de inflação coletadas em 2002, o desvio-padrão das expectativas de inflação para 1, 2, 3 e 4 anos à frente se refere à projeção do mercado para a inflação para os anos 2003, 2004, 2005 e 2006, respectivamente, enquanto que para os dados coletados em 2013, as séries equivalem à projeção de mercado para 2014, 2015, 2016 e 2017. Por sua vez, utilizamos como medida de inflação a inflação acumulada em 12 meses do IPCA, o IPCA mensal, o núcleo da inflação ${ }^{7}$ acumulado em 12 meses e o núcleo da inflação mensal do IPCA. Caso as expectativas de inflação tenham sido coletadas no mês de maio, a inflação acumulada em 12 meses se refere ao período de 12 meses que se encerra em maio daquele ano (inclusive). A utilização dessas séries teve o propósito de harmonizar os resultados obtidos nesta seção com os obtidos na seção anterior. Adicionalmente, a utilização do núcleo de inflação teve o objetivo de analisar

\footnotetext{
${ }^{6}$ Agradeço ao parecerista por indicar este ponto.

${ }^{7}$ A série de núcleo da inflação mensal foi retirada do IPEADATA, e corresponde ao núcleo de médias aparadas com suavização do IPCA.
} 
Tabela 8: IPCA em 12 meses - Critérios de seleção dos modelos

\begin{tabular}{lcccccccc}
\hline & Garch(1,0) & Garch(0,1) & Garch(1,1) & Garch(2,2) & Garch(2,1) & Garch(1,2) & $\operatorname{Tarch}(1,1)$ & $\operatorname{EGarch}(1,1)$ \\
\hline $\mathrm{R}^{2}$ & 0.98 & 0.98 & 0.98 & 0.98 & 0.98 & 0.98 & 0.98 & 0.98 \\
Erro-padrão da regressão & 0.32 & 0.32 & 0.32 & 0.31 & 0.32 & 0.32 & 0.32 & 0.31 \\
Log-verossimilhança & -11.00 & -10.76 & -13.60 & -6.88 & -8.71 & -13.40 & -11.68 & 0.85 \\
Critério de Akaike & 0.23 & 0.21 & 0.26 & 0.20 & 0.21 & 0.27 & 0.25 & 0.12 \\
Critério de Schwarz & 0.39 & 0.36 & 0.42 & 0.40 & 0.39 & 0.45 & 0.43 & 0.32 \\
Critério de Hannan-Quinn & 0.29 & 0.27 & 0.32 & 0.28 & 0.29 & 0.34 & 0.32 & 0.20 \\
\hline
\end{tabular}


Tabela 9: Testes de causalidade de Granger entre a taxa de inflação mensal do IPCA e a volatilidade do modelo GARCH (Proxy da incerteza inflacionária)

\begin{tabular}{|c|c|c|c|c|c|}
\hline \multirow{2}{*}{ Equação } & \multirow{2}{*}{ Variável Excluída } & \multicolumn{2}{|c|}{$\operatorname{GARCH}(1,1)$} & \multicolumn{2}{|c|}{$\operatorname{GARCH}(2,2)$} \\
\hline & & Chi2 & P-valor & Chi2 & P-valor \\
\hline IPCA mensal & Volatilidade condicional & 10.33 & 0.03 & 34.095 & 0.33 \\
\hline Volatilidade condicional & IPCA mensal & 117.46 & 0.00 & 105.04 & 0.00 \\
\hline \multirow{2}{*}{ Equação } & \multirow{2}{*}{ Variável Excluída } & \multicolumn{2}{|c|}{ GARCH $(1,1)$} & \multicolumn{2}{|c|}{$\operatorname{GARCH}(2,2)$} \\
\hline & & Chi2 & P-valor & Chi2 & P-valor \\
\hline IPCA mensal & Volatilidade condicional & 11.26 & 0.02 & 19.13 & 0.00 \\
\hline Volatilidade condicional & IPCA mensal & 122.83 & 0.00 & 74.47 & 0.00 \\
\hline \multirow{2}{*}{ Equação } & \multirow{2}{*}{ Variável Excluída } & \multicolumn{2}{|c|}{ EGARCH $(1,1)$} & \multicolumn{2}{|c|}{ TARCH $(1,1)$} \\
\hline & & Chi2 & P-valor & Chi2 & P-valor \\
\hline IPCA mensal & Volatilidade condicional & 20.73 & 0.000 & 17.64 & 0.00 \\
\hline Volatilidade condicional & IPCA mensal & 173.19 & 0.000 & 169.34 & 0.00 \\
\hline
\end{tabular}

Nota: O teste apresentado é o de Wald, cuja hipótese nula é de que todos os coeficientes defasados de uma variável são conjuntamente iguais a zero na equação da outra variável no VAR.

Tabela 10: Testes de auto-correlação serial dos modelos VAR bivariados estimados (taxa de inflação mensal e volatilidade condicional)

\begin{tabular}{lccc}
\hline Modelo da volatilidade condicional & Defasagens do VAR & Chi2 & Prob $>$ Chi2 \\
\hline GARCH $(1,1)$ & 4 & 5.54 & 0.23 \\
GARCH $(2,2)$ & 3 & 5.85 & 0.21 \\
GARCH $(1,2)$ & 4 & 6.61 & 0.15 \\
GARCH $(2,1)$ & 3 & 3.62 & 0.45 \\
EGARCH $(1,1)$ & 4 & 5.02 & 0.28 \\
TARCH $(1,1)$ & 4 & 6.68 & 0.15 \\
\hline
\end{tabular}

Nota:O teste apresentado é o de multiplicadores de Lagrange, cuja hipótese nula é a de ausência de auto-correlação serial na respectiva defasagem.

a relação entre choques de incerteza inflacionária e o componente permanente da inflação, excluído o efeito de choques.

Para evitar saltos na passagem de um ano para o outro, construiu-se séries de desvio-padrão das expectativas para prazos fixos, de 1 a 4 anos. O procedimento efetuado é descrito a seguir.

A partir da série diária do desvio-padrão das expectativas de inflação para um determinado ano, tirou-se a média de todas as observações em um determinado mês para um determinado ano à frente. Como no transcorrer de um determinado ano as expectativas de inflação acabam se encurtando à razão de $1 / 12$ avos por mês, o passo seguinte foi efetuar uma média ponderada das expectativas de dois anos adjacentes, com os pesos sendo dados pela fração transcorrida de um determinado ano. Assim, na medida em que um determinado ano se aproxima do final, menos peso é dado para o desvio-padrão das expectativas de inflação daquele ano, em comparação com o ano subsequente. Matematicamente, a fórmula é semelhante à adotada em Minella et al. (2003):

$$
D P_{1 j}=\frac{(13-j)}{12} d p_{t}+\frac{(j-1)}{12} d p_{t+1}
$$


Tabela 11: Testes de causalidade de Granger entre a taxa de inflação mensal do IPCA e a volatilidade do modelo GARCH (Proxy da incerteza inflacionária)

\begin{tabular}{|c|c|c|c|c|c|}
\hline \multirow{2}{*}{ Equação } & \multirow{2}{*}{ Variável Excluída } & \multicolumn{2}{|c|}{$\operatorname{GARCH}(1,1)$} & \multicolumn{2}{|c|}{$\operatorname{GARCH}(2,2)$} \\
\hline & & Chi2 & P-valor & Chi2 & P-valor \\
\hline IPCA 12 meses & Volatilidade condicional & 8.03 & 0.04 & 13.61 & 0.00 \\
\hline Volatilidade condicional & IPCA $12 \mathrm{~m}$ & 59.19 & 0.00 & 39.31 & 0.00 \\
\hline \multirow{2}{*}{ Equação } & \multirow{2}{*}{ Variável Excluída } & \multicolumn{2}{|c|}{$\operatorname{GARCH}(1,1)$} & \multicolumn{2}{|c|}{$\operatorname{GARCH}(2,2)$} \\
\hline & & Chi2 & P-valor & Chi2 & P-valor \\
\hline \multirow{2}{*}{$\begin{array}{l}\text { IPCA } 12 \text { meses } \\
\text { Volatilidade condicional }\end{array}$} & Volatilidade condicional & 10.48 & 0.03 & 12.89 & 0.01 \\
\hline & IPCA $12 \mathrm{~m}$ & 60.55 & 0.00 & 47.41 & 0.00 \\
\hline \multirow{2}{*}{ Equação } & \multirow{2}{*}{ Variável Excluída } & \multicolumn{2}{|c|}{$\operatorname{EGARCH}(1,1)$} & \multicolumn{2}{|c|}{$\operatorname{TARCH}(1,1)$} \\
\hline & & Chi2 & P-valor & Chi2 & P-valor \\
\hline IPCA 12 meses & Volatilidade condicional & 31.97 & 0.00 & 10.90 & 0.02 \\
\hline Volatilidade condicional & IPCA $12 \mathrm{~m}$ & 104.14 & 0.00 & 94.09 & 0.00 \\
\hline
\end{tabular}

Nota: O teste apresentado é o de Wald, cuja hipótese nula é de que todos os coeficientes defasados de uma variável são conjuntamente iguais a zero na equação da outra variável no VAR.

Tabela 12: Testes de auto-correlação serial dos modelos VAR bivariados estimados (IPCA acumulado em 12 meses e volatilidade condicional)

\begin{tabular}{|c|c|c|c|}
\hline Modelo da volatilidade condicional & Defasagens do VAR & Chi2 & Prob $>$ Chi 2 \\
\hline $\operatorname{GARCH}(1,1)$ & 3 & 7.93 & 0.09 \\
\hline $\operatorname{GARCH}(2,2)$ & 4 & 4.76 & 0.31 \\
\hline $\operatorname{GARCH}(1,2)$ & 4 & 3.95 & 0.41 \\
\hline $\operatorname{GARCH}(2,1)$ & 4 & 4.92 & 0.29 \\
\hline EGARCH $(1,1)$ & 5 & 0.68 & 0.95 \\
\hline TARCH $(1,1)$ & 4 & 4.98 & 0.28 \\
\hline
\end{tabular}

Nota:O teste apresentado é o de multiplicadores de Lagrange, cuja hipótese nula é a de ausência de auto-correlação serial na respectiva defasagem.

em que $j$ se refere ao mês em que as expectativas de inflação foram coletadas, $d p_{t}$ se refere ao desvio-padrão das expectativas de inflação para o ano t. Assim, a título de exemplo, para o mês de novembro de 2001 dispomos de dados sobre o desvio-padrão das expectativas de inflação para 2001(ano corrente) até 2005 (4 anos à frente). Pela fórmula acima, $t=11$, de forma que o desvio-padrão das expectativas de inflação para 2001 tem peso de 0,17, enquanto o desvio-padrão das expectativas de inflação para 2002 tem peso de 0,83 no cálculo da série de desvio-padrão das expectativas de inflação para 1 ano à frente.

Para a construção de uma série de prazo fixo para os demais anos, o mesmo procedimento foi empregado, utilizando pares adjacentes de anos, uma vez que em janeiro de um determinado ano dispomos de informações para as expectativas de inflação para 4 anos à frente (em janeiro de 2002 dispomos da expectativa para 2006, com prazo real de $5 \operatorname{anos}^{8}$, mas em dezembro de 2002 a expectativa para 2006 tem um prazo real de 4,08 anos). Em janeiro de 2003

\footnotetext{
${ }^{8}$ Como a projeção de inflação é feita para o ano fechado, embora o dado disponível seja para
} 
Tabela 13: Correlações entre inflação e volatilidade (proxy da incerteza inflacionária)

\begin{tabular}{lcc}
\hline & IPCA 12 meses & IPCA mensal \\
\hline $\operatorname{Garch}(1,1)$ & 0,70 & 0,45 \\
$\operatorname{Garch}(2,2)$ & 0,39 & 0,48 \\
$\operatorname{Garch}(2,1)$ & 0,35 & 0,43 \\
$\operatorname{Garch}(1,2)$ & 0,67 & 0,47 \\
$\operatorname{EGarch}(1,1)$ & 0,72 & 0,57 \\
$\operatorname{Tarch}(1,1)$ & 0,66 & 0,54 \\
\hline
\end{tabular}

já dispomos de dados para a expectativa para 2007, com prazo real de 5 anos novamente, sendo incorporados no cálculo descrito acima. Conforme comentando, esse procedimento foi efetuado de forma a suavizar o movimento da série nas viradas dos anos, principalmente nos meses de janeiro, quando a expectativa para um ano adicional se torna disponível. As séries estão apresentadas na Figura 1. Nota-se uma grande elevação em 2003, e um aumento da incerteza inflacionária de longo prazo a partir de 2010, embora em magnitude consideravelmente inferior à do episódio de $2003 .^{9}$

Sob a hipótese de que um aumento da inflação provoca um aumento da incerteza inflacionária, é de se esperar uma correlação positiva entre a série de inflação acumulada em 12 meses e a variabilidade das expectativas de inflação. A Tabela 14 apresenta a correlação entre a série do IPCA acumulado em 12 meses e cada uma das séries de desvio-padrão das expectativas de inflação. Os coeficientes de correlação são positivos e elevados, mostrando uma forte associação entre o nível da inflação e a incerteza inflacionária. A correlação para 1 ano à frente alcança 0,67 , enquanto os valores para 2, 3 e 4 anos são de 0,72 , 0,77 e 0,83 , respectivamente. ${ }^{10}$ Assim, a correlação cresce quanto mais longo for o horizonte $\mathrm{h}$, de forma a incerteza inflacionária de longo prazo está mais correlacionada com o comportamento da inflação corrente. A correlação com o núcleo da inflação, tanto mensal quanto acumulado em 12 meses, também é elevada, prevalecendo a mesma interpretação de anteriormente, de aumento da correlação quanto mais longo o horizonte da incerteza inflacionária.

Para analisar a relação entre o nível da inflação e a incerteza inflacionária, foram estimados vetores autorregressivos (VARs) entre as 4 séries de inflação

4 anos à frente - nesse caso 2006 em relação à 2002-o prazo real é de 5 anos, uma vez que 2002 é o ano "zero". Nesse sentido, a projeção para 2003 em janeiro de 2002 tem prazo de dois anos. Assim, para o cálculo do desvio-padrão para um prazo fixo de 1 ano consideramos as projeções para 2002 e 2003, para 2 anos as projeções para 2003 e 2004, para 3 anos as projeções para 2004 e 2005 e para 4 anos as projeções para 2005 e 2006 e assim por diante ao longo do tempo. Em janeiro de 2003, a projeção para 2007 se torna disponível e entra no cálculo, saindo o dado para 2002. Consideramos, para o calculo do prazo real, anos de 360 dias, com 30 dias em cada mês.

${ }^{9} \mathrm{O}$ pico da incerteza inflacionária em 2003 , e sua subsequente redução, sugere um importante papel para esta variável em episódios desinflacionários. Naquele ano, a inflação acumulada em 12 meses atingiu 17\% em maio, tendo se reduzido para 5\% em maio de 2004 . Concomitantemente, a incerteza inflacionária para 4 anos à frente naquela ocasião recuou do pico de 1,90\% em novembro de 2002 para 0,59\% em abril de 2004, precedendo a redução da inflação.

${ }^{10}$ Se considerarmos a correlação entre a inflação acumulada em 12 meses até o mês anterior ao que o desvio-padrão das expectativas de inflação foi coletado, também obtemos valores expressivos e elevados. Por exemplo, a correlação entre o IPCA acumulado em 12 meses até o mês t-1 e o desvio-padrão das expectativas de inflação coletado no mês t para 1,2,3 e 4 anos à frente é de 0,58, 0,64, 0,71 e 0,78, respectivamente. Assim como na Tabela 14, os coeficientes de correlação foram computados considerando o período que vai de novembro de 2001 a dezembro de 2003. 
Tabela 14: Correlação entre a inflação e o desvio-padrão das expectativas de inflação

\begin{tabular}{lcccc}
\hline $\begin{array}{l}\text { Desvio-padrão das } \\
\text { expectativas de inflação } \\
\mathrm{h} \text { anos à frente }\end{array}$ & $\begin{array}{c}\text { IPCA acumulado IPCA mensal } \\
\text { em 12 meses }\end{array}$ & $\begin{array}{c}\text { Núcleo da inflação } \\
\text { acumulado em } \\
12 \text { meses }\end{array}$ & $\begin{array}{c}\text { Núcleo da } \\
\text { inflação } \\
\text { (mensal) }\end{array}$ \\
\hline $\mathrm{h}=1$ & 0,67 & 0,75 & 0,5 & 0,78 \\
$\mathrm{~h}=2$ & 0,72 & 0,73 & 0,6 & 0,82 \\
$\mathrm{~h}=3$ & 0,77 & 0,66 & 0,71 & 0,84 \\
$\mathrm{~h}=4$ & 0,83 & 0,6 & 0,75 & 0,83 \\
\hline
\end{tabular}

Nota: Os coeficientes de correlação foram calculados considerando o período entre novembro de 2001 e dezembro de 2013.

e o desvio-padrão das expectativas de inflação para 1 a 4 anos à frente. Assim, foram estimados ao todo 16 modelos. A opção pelo modelo VAR foi permitir analisar a relação entre incerteza inflacionária e inflação tratando as duas variáveis como endógenas. Dessa forma, também permitimos que a inflação seja causada pela incerteza inflacionária, além de testar a hipótese tradicional de que a inflação leva a um aumento da incerteza inflacionária.

Os testes de raiz unitária não indicaram a presença de raiz unitária nas séries utilizadas, motivo pelo qual os modelos foram estimados em nível.

Para os 16 modelos, os critérios de informação (AIC, SBIC e Hannan-Quinn) foram utilizados para selecionar as defasagens dos VARs, em geral apontando para três defasagens como a melhor especificação. As séries utilizadas possuem 145 observações, com frequência mensal, de dezembro de 2001 a dezembro de 2013. Os testes dos multiplicadores de Lagrange, apresentados nas Tabelas 16, 18, 20 e 22, não apontaram a presença de autocorrelação serial nos modelos estimados.

As Tabelas 15 e 17 mostram os testes de causalidade de Granger dos modelos estimados, utilizado como medida de inflação o IPCA acumulado em 12 meses e o IPCA mensal, respectivamente. Para os modelos estimados com o IPCA mensal (Tabela 17), em geral encontramos evidência de causalidade de Granger em ambos os sentidos, ie., a incerteza inflacionária Granger-causa a inflação, sendo que a relação em sentido inverso também vale. Entretanto, os resultados são diferentes quando consideramos a incerteza inflacionária de longo prazo, representada pelo desvio-padrão das expectativas de inflação para 3 e 4 anos à frente e a inflação acumulada em 12 meses (Tabela 15). Os testes de causalidade de Granger nos VARs bivariados estimados apontam que a incerteza inflacionária de longo prazo Granger-causa a inflação, enquanto a relação em sentido contrário não vale. O resultado sinaliza a capacidade preditiva da incerteza inflacionária de longo prazo em relação à dinâmica da inflação corrente.

Para os modelos estimados para o núcleo da inflação (mensal e acumulado em 12 meses), apresentados nas Tabelas 19 e 21, os resultados mostram que a incerteza Granger-causa a inflação, com o contrário não valendo. Nesse sentido, a incerteza inflacionária tem capacidade preditiva para explicar o componente permanente da inflação. As funções de impulso-resposta dos VARs, não apresentadas em benefício da concisão, mostram que após um choque ortogonalizado na incerteza inflacionária o núcleo da inflação acumulado em 12 meses se eleva em até 0,4 ponto percentual. Interpretamos esses resultados como um forte indício da importância da ancoragem das expectativas de inflação para a dinâmica da inflação. 
Tabela 15: Testes de causalidade de Granger entre o IPCA acumulado em 12 meses e desvio-padrão das expectativas de inflação (proxy da incerteza inflacionária)

\begin{tabular}{|c|c|c|c|c|c|}
\hline \multirow[t]{2}{*}{ Equação } & \multirow[t]{2}{*}{$\begin{array}{l}\text { Variável } \\
\text { Excluída }\end{array}$} & \multicolumn{2}{|c|}{$\begin{array}{l}\text { Desvio-padrão das } \\
\text { expectativas de inflação } \\
\text { para } 1 \text { ano à frente }\end{array}$} & \multicolumn{2}{|c|}{$\begin{array}{l}\text { Desvio-padrão das } \\
\text { expectativas de inflação } \\
\text { para } 2 \text { anos à frente }\end{array}$} \\
\hline & & Chi2 & P-valor & Chi2 & P-valor \\
\hline IPCA 12 meses & $\begin{array}{l}\text { Desvio-padrão } \\
\text { das expectativas }\end{array}$ & 79.04 & 0.00 & 54.82 & 0.00 \\
\hline $\begin{array}{l}\text { Desvio-padrão } \\
\text { das expectativas }\end{array}$ & IPCA $12 \mathrm{~m}$ & 8.28 & 0.04 & 6.822 & 0.07 \\
\hline \multirow[t]{2}{*}{ Equação } & $\begin{array}{l}\text { Variável } \\
\text { Excluída }\end{array}$ & \multicolumn{2}{|c|}{$\begin{array}{l}\text { Desvio-padrão das } \\
\text { expectativas de inflação } \\
\text { para } 1 \text { ano à frente }\end{array}$} & \multicolumn{2}{|c|}{$\begin{array}{l}\text { Desvio-padrão das } \\
\text { expectativas de inflação } \\
\text { para } 2 \text { anos à frente }\end{array}$} \\
\hline & & Chi2 & P-valor & Chi2 & P-valor \\
\hline IPCA 12 meses & $\begin{array}{l}\text { Desvio-padrão } \\
\text { das expectativas }\end{array}$ & 44.25 & 0.00 & 49.70 & 0.00 \\
\hline $\begin{array}{l}\text { Desvio-padrão } \\
\text { das expectativas }\end{array}$ & IPCA $12 \mathrm{~m}$ & 4.83 & 0.18 & 5.77 & 0.12 \\
\hline
\end{tabular}

Nota: O teste apresentado é o de Wald, cuja hipótese nula é de que todos os coeficientes defasados de uma variável são conjuntamente iguais a zero na equação da outra variável no VAR.

Tabela 16: Testes de auto-correlação serial dos modelos VAR bivariados estimados (IPCA acumulado em 12 meses e desvio-padrão das expectativas de inflação)

Horizonte do desvio-padrão das expectativas de Defasagens do VAR Chi2 Prob $>$ Chi2 inflação

\begin{tabular}{llll}
\hline 1 ano à frente & 3 & 1.58 & 0.81 \\
2 anos à frente & 3 & 2.21 & 0.69 \\
3 anos à frente & 3 & 1.94 & 0.74 \\
4 anos à frente & 3 & 2.36 & 0.66 \\
\hline
\end{tabular}

Nota: O teste apresentado é o de multiplicadores de Lagrange, cuja hipótese nula é a de ausência de auto-correlação serial na respectiva defasagem.

\subsection{Discussão das medidas de incerteza inflacionária}

A despeito das diferentes metodologias para obter a incerteza inflacionária, a análise da relação entre incerteza e inflação produz resultados comuns.

O primeiro resultado é a correlação positiva e elevada entre o nível da inflação e a incerteza inflacionária, conforme apresentado nas Tabelas 13 e 14 .

No caso da incerteza oriunda dos modelos GARCH, obtemos correlações da ordem de 0,4 a 0,7, dependendo de qual modelo da família GARCH é considerado. Para a incerteza inflacionária mensurada a partir do desvio-padrão das expectativas de inflação, os resultados são ainda mais expressivos, com correlações superiores a 0,7 . Outra característica dessa segunda medida é que a correlação entre o nível da inflação e a incerteza cresce com o horizonte da incerteza, no sentido de que a incerteza inflacionária de longo prazo ( 3 a 4 anos à frente) é mais correlacionada com a inflação corrente, quanto mensurada pelo IPCA acumulado em 12 meses.

Um segundo resultado comum às duas medidas é que em geral a inflação Granger-causa a incerteza, com a relação em sentido contrário também 
Tabela 17: Testes de causalidade de Granger entre a inflação mensal e desvio-padrão das expectativas de inflação (proxy da incerteza inflacionária)

\begin{tabular}{|c|c|c|c|c|c|}
\hline \multirow[t]{2}{*}{ Equação } & \multirow[t]{2}{*}{$\begin{array}{l}\text { Variável } \\
\text { Excluída }\end{array}$} & \multicolumn{2}{|c|}{$\begin{array}{l}\text { Desvio-padrão das } \\
\text { expectativas de inflação } \\
\text { para } 1 \text { ano à frente }\end{array}$} & \multicolumn{2}{|c|}{$\begin{array}{l}\text { Desvio-padrão das } \\
\text { expectativas de inflação } \\
\text { para } 2 \text { anos à frente }\end{array}$} \\
\hline & & Chi2 & P-valor & Chi2 & P-valor \\
\hline IPCA mensal & $\begin{array}{l}\text { Desvio-padrão } \\
\text { das expectativas }\end{array}$ & 148.91 & 0.00 & 112.63 & 0.00 \\
\hline $\begin{array}{l}\text { Desvio-padrão } \\
\text { das expectativas }\end{array}$ & IPCA mensal & 10.98 & 0.02 & 3.21 & 0.52 \\
\hline \multirow[t]{2}{*}{ Equação } & $\begin{array}{l}\text { Variável } \\
\text { Excluída }\end{array}$ & \multicolumn{2}{|c|}{$\begin{array}{l}\text { Desvio-padrão das } \\
\text { expectativas de inflação } \\
\text { para } 3 \text { anos à frente }\end{array}$} & \multicolumn{2}{|c|}{$\begin{array}{l}\text { Desvio-padrão das } \\
\text { expectativas de inflação } \\
\text { para } 4 \text { anos à frente }\end{array}$} \\
\hline & & Chi2 & P-valor & Chi2 & P-valor \\
\hline IPCA mensal & $\begin{array}{l}\text { Desvio-padrão } \\
\text { das expectativas }\end{array}$ & 84.47 & 0.00 & 83.73 & 0.00 \\
\hline $\begin{array}{l}\text { Desvio-padrão } \\
\text { das expectativas }\end{array}$ & IPCA mensal & 7.60 & 0.10 & 14.77 & 0.01 \\
\hline
\end{tabular}

Nota: O teste apresentado é o de Wald, cuja hipótese nula é de que todos os coeficientes defasados de uma variável são conjuntamente iguais a zero na equação da outra variável no VAR.

Tabela 18: Testes de auto-correlação serial dos modelos VAR bivariados estimados (IPCA mensal e desvio-padrão das expectativas de inflação)

Horizonte do desvio-padrão das expectativas de Defasagens do VAR Chi2 Prob>Chi2 inflação

\begin{tabular}{llll}
\hline 1 ano à frente & 4 & 2.48 & 0.64 \\
2 anos à frente & 4 & 3.57 & 0.46 \\
3 anos à frente & 4 & 7.76 & 0.10 \\
4 anos à frente & 5 & 4.53 & 0.33 \\
\hline
\end{tabular}

Nota:O teste apresentado é o de multiplicadores de Lagrange, cuja hipótese nula é a de ausência de auto-correlação serial na respectiva defasagem.

valendo na maioria dos casos. Nesse sentido, os resultados são coerentes tanto com a hipótese de Friedman-Ball quanto com a hipótese de CukiermanMeltzer. Para a medida de incerteza mensurada a partir da dispersão das expectativas de inflação, os resultados apontam que a incerteza de longo prazo (3 e 4 anos à frente) tem capacidade preditiva para a inflação, enquanto o contrário não vale. Adicionalmente, a incerteza de longo prazo precede temporalmente o componente permanente da inflação (núcleo da inflação), conforme encontrado nos testes de causalidade de Granger.

A análise do coeficiente de correlação entre as séries de volatilidade da inflação e do desvio-padrão das expectativas de inflação mostra que as duas medidas têm correlação positiva e relativamente elevada, em geral entre 0,5 e 0,6, conforme dados apresentados na Tabela 23.

\section{Conclusão}

Este artigo buscou analisar a relação entre inflação e incerteza inflacionária no Brasil. Resultados obtidos para grande número de países mostram que um aumento da inflação resulta em uma elevação da incerteza inflacionária. Em 
Tabela 19: Testes de causalidade de Granger entre o núcleo da inflação e desviopadrão das expectativas de inflação (proxy da incerteza inflacionária)

\begin{tabular}{|c|c|c|c|c|c|}
\hline \multirow[t]{2}{*}{ Equação } & \multirow[t]{2}{*}{$\begin{array}{l}\text { Variável } \\
\text { Excluída }\end{array}$} & \multicolumn{2}{|c|}{$\begin{array}{l}\text { Desvio-padrão das } \\
\text { expectativas de inflação } \\
\text { para } 1 \text { ano à frente }\end{array}$} & \multicolumn{2}{|c|}{$\begin{array}{l}\text { Desvio-padrão das } \\
\text { expectativas de inflação } \\
\text { para } 2 \text { anos à frente }\end{array}$} \\
\hline & & Chi2 & P-valor & Chi2 & P-valor \\
\hline $\begin{array}{l}\text { Núcleo da inflação } \\
\text { (mensal) }\end{array}$ & $\begin{array}{l}\text { Desvio-padrão } \\
\text { das expectativas }\end{array}$ & 55.28 & 0.00 & 59.13 & 0.00 \\
\hline $\begin{array}{l}\text { Desvio-padrão } \\
\text { das expectativas }\end{array}$ & $\begin{array}{l}\text { Núcelo da inflação } \\
\text { (mensal) }\end{array}$ & 5.40 & 0.06 & 2.65 & 0.26 \\
\hline \multirow[t]{2}{*}{ Equação } & $\begin{array}{l}\text { Variável } \\
\text { Excluída }\end{array}$ & \multicolumn{2}{|c|}{$\begin{array}{l}\text { Desvio-padrão das } \\
\text { expectativas de inflação } \\
\text { para } 3 \text { anos à frente }\end{array}$} & \multicolumn{2}{|c|}{$\begin{array}{l}\text { Desvio-padrão das } \\
\text { expectativas de inflação } \\
\text { para } 4 \text { anos à frente }\end{array}$} \\
\hline & & Chi2 & P-valor & Chi2 & P-valor \\
\hline $\begin{array}{l}\text { Núcleo da inflação } \\
\text { (mensal) }\end{array}$ & $\begin{array}{l}\text { Desvio-padrão } \\
\text { das expectativas }\end{array}$ & 58.33 & 0.00 & 64.45 & 0.00 \\
\hline $\begin{array}{l}\text { Desvio-padrão } \\
\text { das expectativas }\end{array}$ & $\begin{array}{l}\text { Núcelo da inflação } \\
\text { (mensal) }\end{array}$ & 2.75 & 0.43 & 3.21 & 0.20 \\
\hline
\end{tabular}

Nota: O teste apresentado é o de Wald, cuja hipótese nula é de que todos os coeficientes defasados de uma variável são conjuntamente iguais a zero na equação da outra variável no VAR.

Tabela 20: Testes de auto-correlação serial dos modelos VAR bivariados estimados (núcelo da inflação e desvio-padrão das expectativas de inflação)

Horizonte do desvio-padrão das expectativas de Defasagens do VAR Chi2 Prob $>$ Chi2 inflação

\begin{tabular}{llll}
\hline 1 ano à frente & 2 & 5.75 & 0.21 \\
2 anos à frente & 2 & 4.86 & 0.30 \\
3 anos à frente & 3 & 6.87 & 0.14 \\
4 anos à frente & 2 & 7.26 & 0.12 \\
\hline
\end{tabular}

Nota: O teste apresentado é o de multiplicadores de Lagrange, cuja hipótese nula é a de ausência de auto-correlação serial na respectiva defasagem.

um primeiro instante, foi realizada a análise mais comumente encontrada na literatura que relaciona o nível da inflação com incerteza inflacionária. Essa análise estimou modelos da família GARCH, tanto simétricos quanto assimétricos, para a variância da taxa de inflação. A volatilidade condicional então obtida é usualmente tratada na literatura como uma proxy da incerteza inflacionária. Encontramos evidências de causalidade de Granger em ambos os sentidos entre incerteza e inflação.

Em uma segunda etapa, utilizou-se como medida de incerteza inflacionária o desvio-padrão das expectativas de inflação, que tem como vantagem o fato de ser diretamente observável, ao contrário de medidas de incerteza inflacionária oriundas da aplicação de modelos GARCH.

Mostrou-se que essa medida de incerteza inflacionária é bastante correlacionada com a inflação, e que essa relação é mais forte quanto mais longo for o prazo das expectativas de inflação, revelando a maior importância, em termos relativos, da incerteza inflacionária de longo prazo em relação à de curto prazo. Conforme apontado por Kontonikas (2004), a incerteza inflacionária de longo prazo tem maior efeito sobre as decisões intertemporais dos agentes. 
Tabela 21: Testes de causalidade de Granger entre o núcleo da inflação em 12 meses e desvio-padrão das expectativas de inflação (proxy da incerteza inflacionária)

\begin{tabular}{|c|c|c|c|c|c|}
\hline \multirow[t]{2}{*}{ Equação } & \multirow[t]{2}{*}{$\begin{array}{l}\text { Variável } \\
\text { Excluída }\end{array}$} & \multicolumn{2}{|c|}{$\begin{array}{l}\text { Desvio-padrão das } \\
\text { expectativas de inflação } \\
\text { para } 3 \text { anos à frente }\end{array}$} & \multicolumn{2}{|c|}{$\begin{array}{l}\text { Desvio-padrão das } \\
\text { expectativas de inflação } \\
\text { para } 4 \text { anos à frente }\end{array}$} \\
\hline & & Chi2 & P-valor & Chi2 & P-valor \\
\hline $\begin{array}{l}\text { Núcleo da inflação } \\
\text { (12 meses) }\end{array}$ & $\begin{array}{l}\text { Desvio-padrão } \\
\text { das expectativas }\end{array}$ & 33.56 & 0.00 & 42.38 & 0.00 \\
\hline $\begin{array}{l}\text { Desvio-padrão } \\
\text { das expectativas }\end{array}$ & $\begin{array}{l}\text { Núcelo da inflação } \\
\text { (12 meses) }\end{array}$ & 1.49 & 0.68 & 3.25 & 0.35 \\
\hline
\end{tabular}

Nota: O teste apresentado é o de Wald, cuja hipótese nula é de que todos os coeficientes defasados de uma variável são conjuntamente iguais a zero na equação da outra variável no VAR.

Tabela 22: Testes de auto-correlação serial dos modelos VAR bivariados estimados (núcelo da inflação em 12 meses e desvio-padrão das expectativas de inflação)

Horizonte do desvio-padrão das expectativas de Defasagens do VAR Chi2 Prob $>$ Chi2 inflação

\begin{tabular}{llll}
\hline 1 ano à frente & 3 & 6.02 & 0.19 \\
2 anos à frente & 3 & 4.20 & 0.37 \\
3 anos à frente & 3 & 2.16 & 0.70 \\
4 anos à frente & 3 & 0.85 & 0.93 \\
\hline
\end{tabular}

Nota: O teste apresentado é o de multiplicadores de Lagrange, cuja hipótese nula é a de ausência de auto-correlação serial na respectiva defasagem.

Tabela 23: Matriz de correlação entre diferentes medidas de incerteza inflacionária

\begin{tabular}{|c|c|c|c|c|c|c|c|}
\hline & DP1 DP2 & DP3 & DP4 & DP1 & $\mathrm{DP} 2$ & DP3 & DP4 \\
\hline & \multicolumn{3}{|c|}{ IPCA mensal } & \multicolumn{4}{|c|}{ IPCA acumulado em 12 meses } \\
\hline $\operatorname{Garch}(1,1)$ & $0,56 \quad 0,57$ & 0,51 & 0,45 & 0,58 & 0,61 & 0,62 & 0,70 \\
\hline $\operatorname{Garch}(2,2)$ & $0,57 \quad 0,58$ & 0,51 & 0,47 & 0,54 & 0,55 & 0,52 & 0,52 \\
\hline $\operatorname{Garch}(2,1)$ & $0,590,58$ & 0,55 & 0,48 & 0,49 & 0,50 & 0,45 & 0,46 \\
\hline $\operatorname{Garch}(1,2)$ & $0,58 \quad 0,59$ & 0,53 & 0,46 & 0,57 & 0,61 & 0,61 & 0,68 \\
\hline $\operatorname{EGarch}(1,1)$ & $0,73 \quad 0,73$ & 0,67 & 0,64 & 0,56 & 0,61 & 0,69 & 0,75 \\
\hline $\operatorname{Tarch}(1,1)$ & $0,660,66$ & 0,59 & 0,55 & 0,60 & 0,62 & 0,61 & 0,69 \\
\hline
\end{tabular}

Outra evidência nesse sentido é que a incerteza Granger-causa o núcleo da inflação, ou seja, o componente permanente da inflação, conforme apresentado nas tabelas 19 e 21. A maior correlação da incerteza inflacionária de longo prazo com a inflação corrente também está em consonância com o resultado encontrado por Ball \& Cecchetti (1990), de que a relação entre o nível da inflação e a variância dos choques permanentes é maior do que a correlação com os choques temporários.

Adicionalmente, testes de causalidade de Granger a partir de modelos VAR mostram que a incerteza inflacionária de longo prazo (medida pelo desviopadrão das expectativas de inflação) auxilia na previsão da inflação, ao passo que o contrário não vale.

Por último, compararam-se as diferentes medidas de incerteza inflacionária utilizadas, concluindo que ambas são moderadamente correlacionadas. 
Esta pesquisa apresenta, portanto, algumas contribuições ao entendimento da relação entre inflação e incerteza inflacionária no Brasil, suprindo uma lacuna na literatura. Apesar de não ser o primeiro trabalho a tratar do tema para o Brasil, é o primeiro a analisar a relação entre o nível da inflação e a incerteza inflacionária utilizando dados para o período posterior à implantação do regime de metas para a inflação, apresentando assim novas evidências, em adição às pesquisas de Issler (1991) e Vale (2004).

Em comparação com esses estudos, a abordagem adotada neste trabalho tem a vantagem de analisar a relação entre inflação e incerteza inflacionária considerando defasagens adicionais das variáveis, que potencialmente podem ser informativas sobre a relação em questão. Issler (1991) testa o coeficiente da primeira defasagem da inflação na incerteza inflacionária dada pelo GARCH, enquanto que em Vale (2004), a volatilidade condicional da inflação do modelo $\operatorname{GARCH}(1,1)$ aparece na equação da média da inflação e da produção industrial. Como nesta pesquisa os modelos VAR foram estimados com a série de volatilidade condicional dos modelos GARCH e a inflação, permitiu-se uma relação potencialmente mais rica da relação entre as duas variáveis, de forma que defasagens mais longas das duas variáveis se relacionassem entre si, tendo em vista que a maioria dos VARs foi estimada com 3 ou 4 defasagens. Uma contribuição adicional desta pesquisa se refere à análise do papel do desvio-padrão das expectativas de inflação como medida de incerteza inflacionária para o Brasil, uma vez que estudos c onsiderando essa medida tiveram como foco o caso americano, como em Golob (1994) e Holland (1995).

Os resultados não permitem rejeitar de forma clara a hipótese de FriedmanBall ou a hipótese de Cukierman-Meltzer, apresentado evidências favoráveis a ambas. À luz da evidência internacional, os resultados estão em linha com os resultados encontrados para os EUA (Ball \& Cecchetti (1990); Golob (1994); Holland (1995)), no Reino Unido (Kontonikas 2004), para diversos países europeus (Caporale et al. 2012) e para o G7 (Berument \& Dincer 2005, Grier \& Perry. 1998), no sentido de que a inflação no Brasil Granger-causa a incerteza inflacionária. Estudos anteriores sobre o tema apontaram resultados favoráveis à hipótese de Friedman-Ball (Issler 1991) ou Cukierman-Meltzer (Vale 2004) para o Brasil. Diante dos resultados encontrados, a julgar pelo passado recente, compreendendo o período do regime de metas para inflação, a ancoragem das expectativas de inflação é essencial para uma dinâmica benigna da inflação corrente, que por sua vez reforça a ancoragem das expectativas.

\section{Agradecimentos}

Agradeço o editor, Eliezer Martins Diniz, e os pareceristas anônimos.

\section{Referências Bibliográficas}

Ball, L. (1992), 'Why does high inflation raise inflation uncertainty?', Journal of Monetary Economics (29), 371-388.

Ball, L. \& Cecchetti, S. G. (1990), 'Inflation and uncertainty at short and long horizons', Brookings Papers on Economic Activity 1, 215-254.

Barro, R. \& Gordon, D. (1983), 'A positive theory of monetary policy in a natural rate model', J. Polit. Econ (91), 589-610. 
Berument, H. \& Dincer, N. (2005), 'Inflation and inflation uncertainty in the g-7 countries', Physica A (348), 371-379.

Bollerslev, T. (1986), 'Generalized autoregressive conditional heteroskedasticity', Journal of Econometrics (31), 307-327.

Caporale, G. M., Onorante, L. \& Paesani, P. (2012), 'Inflation and inflation uncertainty in the euro area', Empirical Economics .

Cukierman, A. \& Meltzer, A. (1986), 'A theory of ambiguity, credibility, and inflation under discretion and asymmetric information', Econometrica (54), 1099-1128.

Friedman, M. (1976), 'Nobel lecture: Inflation and unemployment'. Acesso em: 18 de julho de 2013.

URL: http://www.nobelprize.org/nobel_prizes/economicsciences/laureates/1976/friedman-lecture.pdf

Golob, J. (1994), 'Does inflation uncertainty increase with inflation?', Federal Reserve Bank of Kansas City Economic Review pp. 27-38.

Grier, K. B. \& Perry., M. J. (1998), 'On inflation and inflation uncertainty in the g-7 countries', Journal of International Money and Finance (17), 671-89.

Holland, A. S. (1984), 'Does higher inflation lead to more uncertain inflation?', Federal Reserve Bank of St. Louis Review pp. 15-26.

Holland, A. S. (1995), 'Inflation and uncertainty: Tests for temporal ordering', Journal of Money, Credit, and Banking 27(3), 827-837.

Issler, J. (1991), 'Inflation level and uncertainty: Evidence using brazilian data', Revista Brasileira de Economia 45(3), 473-482.

Kontonikas, A. (2004), 'Inflation and inflation uncertainty in the united kingdom, evidence from garch modelling', Economic Modelling 21(3), 525-543.

Minella, A., Freitas, P. S., Goldfajn, I. \& Muinhos, M. K. (2003), Inflation targeting in brazil: Constructing credibility under exchange rate volatility. Working Papers Series 77.

Morettin, P. (2006), 'Econometria financeira', ABE-Associação Brasileira de Estatística .

Okun, A. M. (1971), 'The mirage of steady inflation', Brookings Papers on Economic Activity pp. 485-498.

Vale, S. (2004), 'Inflation, growth and real and nominal uncertainty: Some bivariate garch-in-mean evidence for brazil', Revista Brasileira de Economia . 


\section{Apêndice A}

Este apêndice apresenta os testes de raiz unitária empregados nas séries utilizadas: IPCA acumulado em 12 meses, desvio-padrão das expectativas de inflação para 1 a 4 anos à frente e volatilidades condicionais geradas pelos modelos GARCH estimados.

O teste apresentado é denominado de Dick-Fuller modificado, onde a série de tempo a ser testada primeiramente é transformada por meio do procedimento de mínimos quadrados generalizados. A série a ser testada é regredida em uma constante e uma tendência linear, e aplica-se um teste ADF aos resíduos da regressão. Como as séries utilizadas não aparentam ter uma tendência, a versão do teste utilizada considerou apenas uma constante na primeira etapa, ou seja, foi utilizado o procedimento de GLS demeaning ao invés de GLS adetrending. A principal vantagem deste teste em comparação com os testes de Dick-Fuller aumentado e o teste de Philips-Perron é o seu maior poder. Adicionalmente, os testes ADF e Philips-Perron tendem a rejeitar a hipótese nula com maior frequência. De forma análoga a este teste, o teste de Dick-Fuller modificado consiste em testar a hipótese de que $\beta=0$ na seguinte regressão:

$$
\Delta y_{t}=\alpha+\beta y_{t-1}+\theta_{1} \Delta y_{t-1}+. .+\theta_{k} \Delta y_{t-k}+u_{t}
$$

A hipótese nula do teste efetuado é que $y_{t}$ é um passeio aleatório com média diferente de zero, mas sem tendência, uma vez que a observação visual das séries não aponta para uma tendência nos dados. Para as séries do IPCA acumulado em 12 meses, IPCA ao mês e desvio-padrão das expectativas de inflação rejeitamos a hipótese de raiz unitária por meio do teste DF-GLS.

Em virtude da grande quantidade de séries de volatilidade condicional, apresentamos apenas o teste de Philips-Perron, que aponta para a estacionariedade das séries, com exceção da volatilidade condicional gerada pelo modelo E-GARCH $(1,1)$ para o IPCA acumulado em 12 meses.

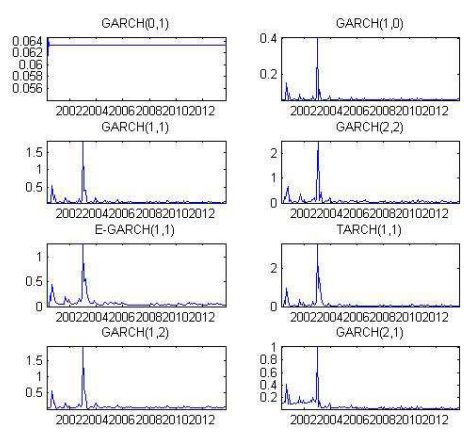

Figura A.1: Volatilidades condicionais geradas por cada modelo utilizando o IPCA acumulado em 12 meses 


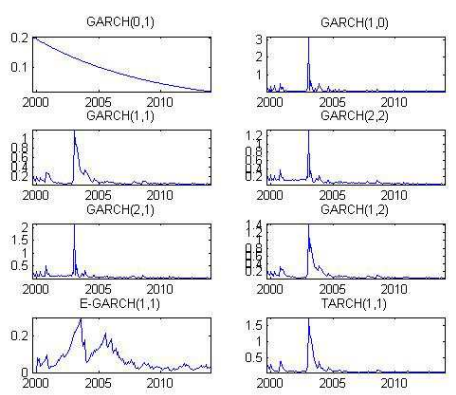

Figura A.2: Volatilidades condicionais geradas por cada modelo utilizando o IPCA mensal

Tabela A.1: Testes de raiz unitária DF-GLS

\begin{tabular}{|c|c|c|c|c|c|c|}
\hline Defasagens & $\begin{array}{c}\text { IPCA } \\
12 \text { meses }\end{array}$ & $\begin{array}{l}\text { Desvio-padrão } \\
1 \text { ano à frente }\end{array}$ & $\begin{array}{l}\text { Desvio-padrão } \\
2 \text { anos à frente }\end{array}$ & $\begin{array}{l}\text { Desvio-padrão } \\
3 \text { anos à frente }\end{array}$ & $\begin{array}{l}\text { Desvio-padrão } \\
4 \text { anos à frente }\end{array}$ & $\begin{array}{l}\text { IPCA } \\
\text { ao mês }\end{array}$ \\
\hline 1 & $-4.128^{* * *}$ & $-6.026^{* * *}$ & $-6.371^{* * *}$ & $-4.027^{* * *}$ & $-3.873^{* * *}$ & $-4.395^{* * *}$ \\
\hline 2 & $-3.745^{* * *}$ & $-6.038^{* * *}$ & $-6.688^{* * *}$ & $-4.632^{* * *}$ & $-3.763^{* * *}$ & $-3.870^{* * *}$ \\
\hline 3 & $-4.440^{* * *}$ & $-5.750^{* * *}$ & $-6.518^{* * *}$ & $-4.652^{* * *}$ & $-3.819^{* * *}$ & $-3.700^{* * *}$ \\
\hline 4 & $-5.051^{* * *}$ & $-5.351^{* * *}$ & $-6.042^{* * *}$ & $-4.656^{* * *}$ & $-5.138^{* * *}$ & $-4.155^{* * *}$ \\
\hline 5 & $-4.415^{* * *}$ & $-5.101^{* * * *}$ & $-6.055^{* * *}$ & $-4.792^{* * * *}$ & $-6.305^{* * *}$ & $-3.875^{* * *}$ \\
\hline 6 & $-4.072^{* * *}$ & $-5.101^{* * *}$ & $-5.554^{* * *}$ & $-3.841^{* * *}$ & $-4.699^{* * *}$ & $-3.824^{* * *}$ \\
\hline 7 & $-3.590^{* * *}$ & $-4.835^{* * *}$ & $-5.563^{* * *}$ & $-4.219^{* * *}$ & $-4.464^{* * *}$ & $-2.898^{* *}$ \\
\hline 8 & $-3.682^{* * *}$ & $-5.516^{* * *}$ & $-6.053^{* * *}$ & $-4.614^{* * *}$ & $-4.576^{* * *}$ & -2.578 \\
\hline 9 & $-3.752^{* * *}$ & $-6.247^{* * *}$ & $-5.856^{* * *}$ & $-3.924^{* * *}$ & $-4.032^{* * *}$ & -2.457 \\
\hline 10 & $-3.489^{* *}$ & $-4.867^{* * *}$ & $-5.044^{* * *}$ & $-3.787^{* * *}$ & $-3.827^{* * *}$ & -2.169 \\
\hline 11 & $-2.843^{* *}$ & $-4.049^{* * *}$ & $-5.079^{* * *}$ & $-3.610^{* * *}$ & $-3.811^{* * *}$ & -2.056 \\
\hline 12 & -2.456 & $-4.162^{* * *}$ & $-5.127^{* * *}$ & $-3.501^{* *}$ & $-3.775^{* * *}$ & -2.434 \\
\hline 13 & $-2.843^{* *}$ & $-4.101^{* * *}$ & $-4.754^{* * *}$ & $-3.436^{* *}$ & $-3.754^{* * *}$ & -2.207 \\
\hline \multicolumn{7}{|c|}{ Defasagens selecionadas por cada critério } \\
\hline Ng-Perron & 13 & 13 & 8 & 9 & 11 & 12 \\
\hline SIC & 13 & 11 & 8 & 8 & 5 & 1 \\
\hline MAIC & 13 & 11 & 13 & 12 & 11 & 10 \\
\hline
\end{tabular}

${ }^{* * *},{ }^{* *}, \mathrm{e}^{*}$ indicam significância estatistica a $1 \%, 5 \%$ e $10 \%$, respectivamente.

Tabela A.2: Testes de raiz unitária - Philips Perron

\begin{tabular}{|c|c|c|}
\hline \multicolumn{3}{|c|}{ Volatilidade condicional IPCA ao mês IPCA acumulado em 12 meses } \\
\hline Modelo & P-valor & P-valor \\
\hline $\operatorname{GARCH}(0,1)$ & 0 & 0 \\
\hline $\operatorname{GARCH}(1,0)$ & 0 & 0 \\
\hline $\operatorname{GARCH}(1,1)$ & 0 & 0.0004 \\
\hline $\operatorname{GARCH}(2,2)$ & 0 & 0 \\
\hline E-GARCH $(1,1)$ & 0 & 0.2238 \\
\hline TARCH $(1,1)$ & 0 & 0.0001 \\
\hline $\operatorname{GARCH}(1,2)$ & 0 & 0 \\
\hline $\operatorname{GARCH}(2,1)$ & 0 & 0 \\
\hline
\end{tabular}

Nota: A tabela apresenta o p-valor do teste, cuja hipótese nula é de que a série possui uma raiz unitária 